\title{
Thank you to our CEAS Space Journal Reviewers
}

\author{
Stefan Leuko ${ }^{1}$
}

Published online: 6 February 2021

(c) The Author(s) 2021

It is well-known that the quality of a scientific journal strongly depends on the rigorous work of the people involved in the review process, such as the Associate Editors, but in particular our esteemed team of Reviewers.

The editors of the CEAS Space Journal highly appreciate the dedication and the cooperation of the Reviewers listed below, who have made their excellent contributions to maintain the high quality of the Journal throughout 2020.

Aboudonia Ahmed
Aglietti Guglielmo
Aguado Fernando
Anisinov Andrei
Anthoine Jerome
Baier Horst
Banyai Tamas
Barbante Paolo
Batonneau Yann
Beck Fabian
Belyaev Andrei
Bennani Samir
Benson Conor
Berend Nicolas
Bevilacqua Riccardo
Bollino Kevin
Borrelli Salvatore
Bouchez Marc
Braxmaier Claus
Caillat Thierry
Carrer Leonardo
Chedevergne François
Coe Graham
Conde Luis
Cowley Aidan
Danzeca Salvatore
Di Clemente Marco

Aboudonia Ahmed

Aglietti Guglielmo

Aguado Fernando

Anisinov Andrei

Anthoine Jerom

Banyai Tamas

Barbante Paolo

Batonneau Yann

Belyaev Andrei

Bennani Samir

Benson Conor

Bevilacqua Riccardo

Bollino Kevin

Borrelli Salvatore

Caillat Thierry

Carrer Leonardo

Chedevergne François

Coe Graham

Conde Luis

Cowley Aidan

Di Clemente Marco

\author{
Dubois-Matra Olivier \\ Dupuis Paul-Eric \\ Duzellier Sophie \\ Ecker Tobias \\ Emanuelli Matteo \\ Ertel Hanno \\ Evain Helene \\ Ewald Ralf \\ Fabries Christophe \\ Famengo Alessia \\ Fateri Miranda \\ Faye Delphine \\ Fdida Nicolas \\ Ferguson Philip \\ Fix Andreas \\ Freudenmann Dominic \\ Frueh Carolin \\ Gaillard Lionel \\ Gernoth Andreas \\ Gil Fernandez Jesus \\ Gouzman Irina \\ Greda Lukasz \\ Grundmann Jan Thimo \\ Gutiérrez Jordi \\ Haddad Emile \\ Hager Philipp \\ Haupt Matthias
}

\author{
Hormigo Tiago \\ Horri Nadjim \\ Horsley Matthew \\ Horstmann Birger \\ Huh Jeongmoo \\ Johnston Mark \\ Jones Harry \\ Joshi Shashikant \\ Karatekin Ozgur \\ Kaschubek Daniel \\ Kazakovtsev Victor \\ Kirschner Volker \\ Koryanov Vsevolod \\ Lafont Ugo \\ Laporte Christophe \\ Lasseur Christophe \\ Lauck Felix \\ Lavagna Michéle \\ Lefort Xavier \\ Lengowski Michael \\ Leone Bruno \\ Lorenz Sven \\ Lueders Caroline \\ Maiwald Volker \\ Martelli Emanuele \\ Mazouffre Stéphane \\ Michaud Véronique
}

Managing Editor CEAS Space Journal.

Stefan Leuko

stefan.leuko@dlr.de

1 Deutsches Zentrum für Luft- und Raumfahrt e.V. (DLR),

Hansestraße 115, D - 51149 Cologne, Germany 


Mignot Jean
Minisci Edmondo
Mueller Vitali
Navarro Medina Fermin
Panerai Francesco
Pavarin Daniele
Pezzella Giuseppe
Pfaab Kilian
Pfitzner Michael
Privat Michel
Puillet Christian
Rampini Riccardo
Rana Loveneesh
Rawson Anthony
Rodrigues Gonçalo
Rohwer Klaus
Rovatti Marco
Ruiz Lander
Runte Torben

Schafer Ewan
Schein Jochen
Schoenherr Tony
Schröder Fritz-Gerald
Schwane Richard
Schwendemann Daniel
Seiler Rene
Shankar Uday
Shi Qinzhong
Sinko John
Smith Walter F
Stark Ralf
Stephenson Keith
Stesina Fabrizio
Tajmar Martin
Tanno Hideyuki
Thoemel Jan
Tran Philippe
Traub Constantin

Tuanjie Li

Valencia Bel Ferran

Van Loon Jack

Vassilis Kostopoulos

Viola Nicole

Voigt Siegfried

Vos Jan

Wagner Norbert

Wang Peiyuan

Wedler Armin

Wiertz Thierry

Wijker Jaap

Wittig Manfred

Woronowicz Michael

Wulf Christina

Zhang Zhe

Zubko Vladislav
Funding Open Access funding enabled and organized by Projekt DEAL.

Open Access This article is licensed under a Creative Commons Attribution 4.0 International License, which permits use, sharing, adaptation, distribution and reproduction in any medium or format, as long as you give appropriate credit to the original author(s) and the source, provide a link to the Creative Commons licence, and indicate if changes were made. The images or other third party material in this article are included in the article's Creative Commons licence, unless indicated otherwise in a credit line to the material. If material is not included in the article's Creative Commons licence and your intended use is not permitted by statutory regulation or exceeds the permitted use, you will need to obtain permission directly from the copyright holder. To view a copy of this licence, visit http:// creativecommons.org/licenses/by/4.0/.

Publisher's Note Springer Nature remains neutral with regard to jurisdictional claims in published maps and institutional affiliations. 